\title{
APLICAÇÃo DE PLANEJAMENTO FATORIAL A PROTOCOLO DE EXTRAÇÃo E FIXAÇÃO DE SULFETOS VOLATILIZÁVEIS POR ACIDIFICAÇÃO (SVA) EM AMOSTRAS DE SEDIMENTO
}

\author{
Danielle Caroline Schnitzler e Marco Tadeu Grassi* \\ Departamento de Química, Universidade Federal do Paraná, CP 19081, 81531-990 Curitiba - PR, Brasil \\ Sueli Pércio Quinaia \\ Departamento de Química, Universidade Estadual do Centro-Oeste, CP 3010, 85015-430 Guarapuava - PR, Brasil
}

Recebido em 13/2/08; aceito em 27/11/08; publicado na web em 11/5/09

\begin{abstract}
FACTORIAL DESIGN USED ON THE EXTRACTION AND FIXATION OF ACID VOLATILE SULFIDES (AVS) FROM SEDIMENT SAMPLES. The AVS is defined operationally as acid volatile sulfide, which is a controlling phase on the partition of some metallic species in sediments. A Factorial design was evaluated by means of 16 experiments and using four variables: temperature, extraction time, $\mathrm{N}_{2}$ flow, and volume of the $\mathrm{S}^{2-}$ collection solution. The factors that contributed to the efficiency of the process were the extraction time and the $\mathrm{N}_{2}$ flow. Trapping of $\mathrm{S}^{2-}$ was efficient in $\mathrm{AAB}$. The $\mathrm{S}^{2-}$ was quantified using a potentiometric procedure. Recovery tests for $\mathrm{S}^{2-}$ concentrations varying from $1 \times 10^{-5}$ to $1 \times 10^{-4} \mathrm{~mol} \mathrm{~L}^{-1}$ were in the range from 93 to $116 \%$.
\end{abstract}

Keywords: factorial design; sediments; AVS.

\section{INTRODUÇÃO}

O sedimento é um importante compartimento do ecossistema aquático, formando uma variedade de ambientes e habitats para diferentes organismos. Além disso, atua no transporte físico, no acúmulo ou liberação de nutrientes e contaminantes servindo, consequentemente, como fonte direta e indireta destes para organismos de toda a cadeia alimentar. Trata-se, portanto, de um compartimento dinâmico onde ocorrem vários processos físicos, químicos e biológicos. Dependendo das condições desses processos ocorre a chamada remobilização de espécies, representando uma fonte de contaminação para todo o corpo aquático. ${ }^{1}$

Entre as espécies que podem sofrer remobilização estão os metais, que podem existir naturalmente no ambiente. Alguns metais são considerados micronutrientes essenciais, tais como cobre e zinco, sendo necessários em pequenas concentrações para a manutenção da saúde dos seres vivos. Entretanto, quando ocorre um aumento dessas concentrações, efeitos deletérios começam a surgir. Existem outros metais que não possuem função biológica conhecida, como cádmio, chumbo e mercúrio. Nesse contexto, o aumento da concentração dos metais tornou-se uma preocupação mundial principalmente como consequência das atividades antrópicas, geradoras de despejos agrícolas, industriais e urbanos que, inevitavelmente, atingem os corpos de água. ${ }^{2}$ A biodisponibilidade de metais em sedimentos pode ser prevista pela teoria do equilíbrio de partição entre as fases sólida e aquosa, ou seja, o sedimento e a água intersticial. Essa teoria considera que, devido à baixa solubilidade dos sulfetos metálicos, os metais podem permanecer imobilizados no sedimento, especialmente sob condições anóxidas. ${ }^{3}$

Os sulfetos pesquisados neste trabalho são os chamados sulfetos volatilizáveis por acidificação (SVA), ou seja, a fração mais lábil associada aos monossulfetos de ferro e manganês mais solúveis. O SVA é, portanto, obtido operacionalmente por uma extração ácida a frio. O procedimento é realizado a frio para não ocorrer a extração do sulfeto mais resistente na forma de pirita. Nesse processo de extração ocorre a volatilização do SVA na forma de $\mathrm{H}_{2} \mathrm{~S}{ }^{4}$

*e-mail: mtgrassi@quimica.ufpr.br
$\mathrm{O} \mathrm{H}_{2} \mathrm{~S}$ é arrastado e em seguida coletado em uma solução alcalina. Variações no preparo da solução alcalina são interessantes devido às características físico-químicas dos sulfetos. Os sulfetos são espécies altamente instáveis, possíveis de sofrer perdas por volatilização ou por oxidação afetando diretamente o sistema de extração e coleta do SVA.

Outros parâmetros importantes no processo de extração e coleta do SVA são a vazão do gás responsável por carrear o $\mathrm{H}_{2} \mathrm{~S}$ da amostra até a solução coletora e o tempo necessário para todo o $\mathrm{H}_{2} \mathrm{~S}$ chegar à solução coletora. Esses parâmetros podem variar conforme a configuração do sistema empregado.

Para avaliação desses parâmetros é interessante empregar um planejamento fatorial, que consiste de uma ferramenta estatística que apresenta muitas vantagens, dentre elas, a simplicidade, a otimização simultânea de todos os fatores envolvidos no sistema, com menor número de experimentos, maior rapidez e principalmente maior eficiência. Por essas vantagens, o planejamento fatorial vem sendo cada vez mais utilizado nas mais diversas áreas para diferentes amostras e propósitos. Dentre os planejamentos fatoriais mais utilizados encontra-se o tipo fatorial $2^{\mathrm{k}}$, onde $k$ corresponde ao número de fatores que são utilizados na otimização. ${ }^{5}$

O objetivo deste estudo foi otimizar o protocolo analítico de extração, fixação e determinação de SVA utilizando $\mathrm{HCl} 6 \mathrm{~mol} \mathrm{~L}^{-1} \mathrm{em}$ matrizes de sedimento provenientes de ambientes aquáticos fluviais de relevância ambiental. Desta forma, foi empregado um planejamento fatorial de $2^{4}$ experimentos, não só para avaliar a significância das variáveis, mas também, para indicar as condições favoráveis para obtenção dos melhores resultados na extração e coleta do SVA.

\section{PARTE EXPERIMENTAL}

Uma solução denominada tampão alcalino antioxidante (TAA) foi preparada para a fixação do SVA. Para isso foram dissolvidos $18 \mathrm{~g}$ de hidróxido de sódio, 16,2 g de ácido ascórbico e $72 \mathrm{~g}$ de salicilato de sódio para $1 \mathrm{~L}$ de água do tipo Milli-Q. O pH desta solução é 13,6. ${ }^{6}$

Um eletrodo do tipo íon-seletivo (EIS) de estado sólido foi utilizado nas determinações do SVA. Este sensor potenciométrico é baseado em uma membrana sólida de $\mathrm{Ag}_{2} \mathrm{~S}$ que é utilizada extensamente para a determinação de íons sulfeto. ${ }^{7}$ Os potenciais do eletrodo são 
inicialmente verificados por uma solução de dicromato de potássio $1 \mathrm{~g} \mathrm{~L}^{-1}$ com potencial definido em $\mathrm{pH} 7,00$ a $25^{\circ} \mathrm{C}$.

Para a verificação da faixa linear do eletrodo redox colocou-se 20 $\mathrm{mL}$ de solução TAA em uma célula eletroquímica, em meio inerte, e foram feitas adições de solução padrão de sulfeto variando-se a concentração na faixa de $1 \times 10^{-8}$ a $1,6 \times 10^{-3} \mathrm{~mol} \mathrm{~L}^{-1}$. Com esse procedimento, foram determinados o limite prático de detecção (LPD) e o limite inferior de resposta linear (LIRL). ${ }^{8}$

Para a preparação da solução padrão estoque de sulfeto pesou-se 2,4018 g de $\mathrm{Na}_{2} \mathrm{~S} \cdot 9 \mathrm{H}_{2} \mathrm{O}$ e dissolveu-se em $100 \mathrm{~mL}$ de solução TAA. Diluições necessárias da solução estoque de sulfeto padrão foram realizadas em TAA no dia de cada experimento.

A concentração da solução padrão de sulfeto de sódio foi determinada por titulação potenciométrica. Uma alíquota de $10 \mathrm{~mL}$ de solução de sulfeto de sódio e $10 \mathrm{~mL}$ de TAA foram transferidos para uma célula eletroquímica onde o eletrodo de $\mathrm{Ag} / \mathrm{Ag}_{2} \mathrm{~S}$ foi inserido, mantendo-se o sistema, não só sob meio inerte, mas também sob agitação magnética. Desta forma, para a obtenção da concentração da solução padrão de sulfeto de sódio o titulante contendo chumbo na concentração $5 \times 10^{-4} \mathrm{~mol} \mathrm{~L}^{-1}$ foi adicionado em incrementos de $0,2 \mathrm{~mL}$. Uma curva típica de titulação foi construída a partir desse experimento. O ponto de equivalência dessa curva foi determinado pelo cálculo da primeira derivada. ${ }^{3,9}$

Testes de recuperação foram realizados para verificar a eficiência do sistema analítico de extração e fixação de SVA na faixa de concentração de $1 \times 10^{-4}$ a $1 \times 10^{-5} \mathrm{~mol} \mathrm{~L}^{-1}$.

O sistema analítico de extração e fixação é mostrado na Figura 1.

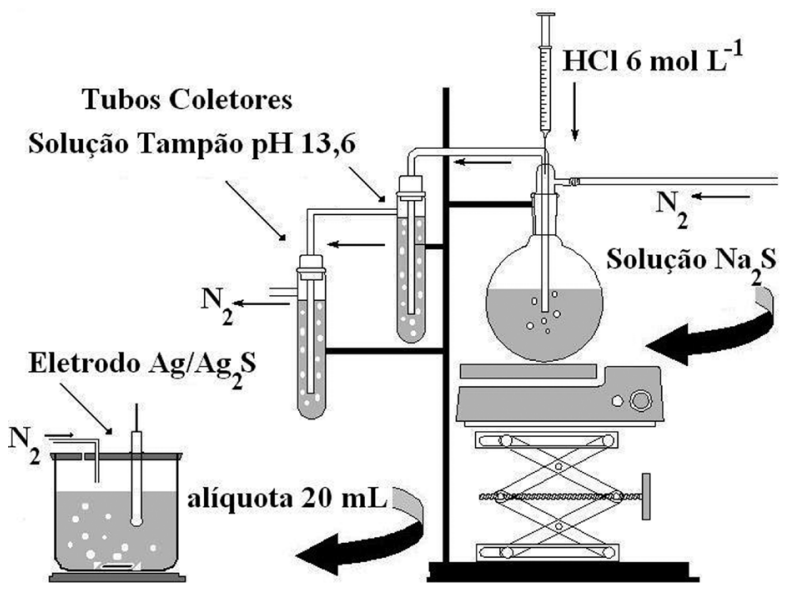

Figura 1. Esquema analítico de extração e fixação de sulfetos

O arranjo consiste de um cilindro de gás nitrogênio e um balão de fundo redondo de $500 \mathrm{~mL}$ conectado a um septo e a dois frascos de $200 \mathrm{~mL}$ para a fixação do SVA. O balão de fundo redondo contém a amostra de sedimento. $O$ gás nitrogênio é passado na suspensão contendo a amostra e, posteriormente, em todo o sistema. Ácido clorídrico é introduzido com uma seringa previamente conectada ao septo. Os frascos contendo a solução TAA estão unidos através de tubos de silicone. ${ }^{4}$

A determinação do SVA foi fundamentada em uma curva de adição de padrão. ${ }^{9}$ Após a extração, foram retirados $20 \mathrm{~mL}$ da solução coletora TAA do primeiro coletor, Figura 1, que contém o SVA retirado da amostra. Esse volume foi transferido para uma célula eletroquímica. Em seguida, o eletrodo foi mergulhado na solução, registrando-se o potencial. Este procedimento foi repetido para a amostra contida no segundo coletor, Figura 1.

Definido todo o sistema experimental do SVA, foi realizado um estudo quimiométrico através de um planejamento fatorial de $2^{4}$ experimentos. Os 16 ensaios foram conduzidos aleatoriamente e as variáveis experimentais foram designadas com o sinal "-" para as variáveis de menor nível e "+" para as variáveis de maior nível. As variáveis foram as seguintes: tempo de extração do SVA (30 ou $60 \mathrm{~min}$ ), vazão de gás nitrogênio (40 ou $90 \mathrm{~cm}^{3} \mathrm{~min}^{-1}$ ), temperatura (ambiente ou $0{ }^{\circ} \mathrm{C}$ ) e volume (100 ou $150 \mathrm{~mL}$ ) de solução TAA.

As determinações de SVA foram realizadas empregando-se amostras de sedimento, coletadas em dois locais nos Rios Iraí e Iguaçu que compõem a Bacia do Alto Iguaçu, na Região Metropolitana de Curitiba (RMC). A Bacia Hidrográfica do Iguaçu é o maior complexo hídrico do estado do Paraná, com uma área de 55.024 km², formado pelo encontro dos Rios Iraí e Atuba na parte leste do município junto à divisa dos municípios de Pinhais e São José dos Pinhais. O ponto de coleta fica a jusante da estação de tratamento de esgoto de Curitiba, a ETE Belém. O ponto de coleta no Rio Iraí localiza-se à montante da cidade de Curitiba e representa um ambiente menos impactado. A localização dos pontos de amostragem na RMC é mostrada na Figura 2. ${ }^{10}$
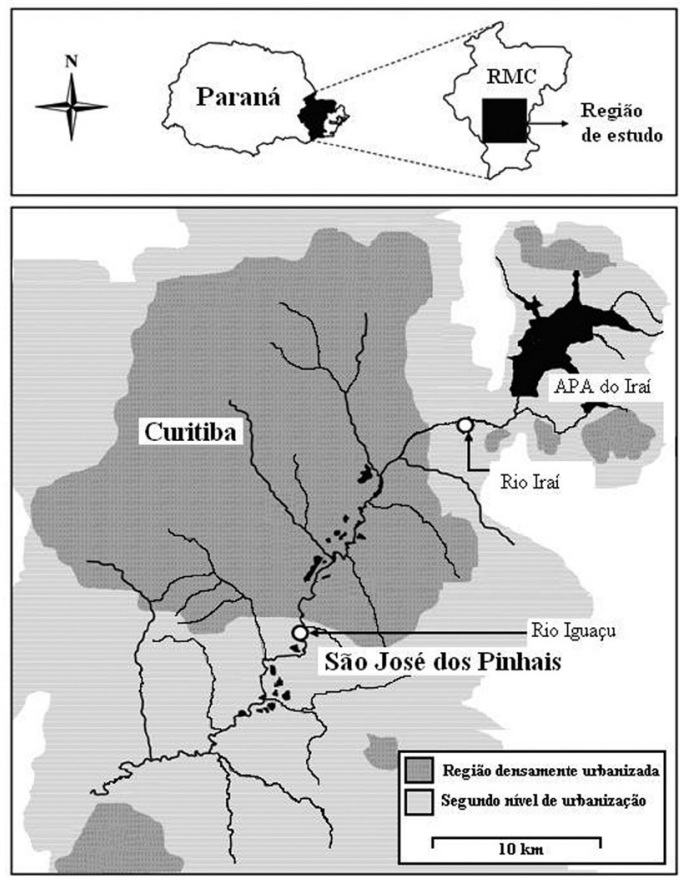

Figura 2. Mapa de localização dos pontos de amostragem

Toda a coleta, preparação e análise das amostras foram conduzidas usando protocolos de técnicas limpas, ${ }^{11}$ de forma a minimizar processos de contaminação.

As amostras foram coletadas através do uso de um testemunhador construído em nosso laboratório, constituído de um tubo de PVC com $4,5 \mathrm{~cm}$ de d. i., 2,22 $\mathrm{m}$ de altura, sendo que 1,13 $\mathrm{m}$ desta altura se referee ao compartimento de coleta. A preservação do testemunho é de grande importância para a determinação da concentração de SVA.

Após a coleta, as amostras foram seladas em sacos plásticos e colocadas em uma caixa refrigerada, mantida à temperatura de $4^{\circ} \mathrm{C}$ até a chegada no laboratório.

Os sedimentos foram manipulados em atmosfera inerte. Aproximadamente 5 a $10 \mathrm{~g}$ de sedimento úmido da camada superficial, 0 a $3 \mathrm{~cm}$, foi colocado em um balão de fundo redondo de $500 \mathrm{~mL}$ de capacidade e adicionou-se $100 \mathrm{~mL}$ de água Milli-Q. A amostra foi purgada por $10 \mathrm{~min}$ com $\mathrm{N}_{2}$ sob vazão contínua de $90 \mathrm{~cm}^{3} \mathrm{~min}^{-1}$. Posteriormente, foram adicionados $20 \mathrm{~mL}$ de $\mathrm{HCl} 6 \mathrm{~mol} \mathrm{~L}^{-1}$ purifica- 
do ${ }^{11}$ introduzidos no balão de reação com o auxílio de uma seringa. Em seguida, gás nitrogênio foi borbulhado por $60 \mathrm{~min}$ a uma vazão de $90 \mathrm{~cm}^{3}$ min $^{-1}$ sob agitação contínua. Foram inseridos $100 \mathrm{~mL}$ de solução TAA em dois tubos para a coleta e fixação do SVA. A reação entre os sulfetos metálicos dissolvidos pelo $\mathrm{HCl}$ gera $\mathrm{H}_{2} \mathrm{~S}$ que é fixado na solução TAA. Após a realização da extração e fixação do SVA, as soluções contidas nos tubos coletores foram quantificadas pela técnica potenciométrica. ${ }^{7,12}$

\section{RESULTADOS E DISCUSSÃO}

$\mathrm{O}$ eletrodo redox de $\mathrm{Ag} / \mathrm{Ag}_{2} \mathrm{~S}$ apresentou uma faixa dinâmica de trabalho entre $3,5 \times 10^{-6}$ e $3,5 \times 10^{-3} \mathrm{~mol} \mathrm{~L}^{-1}$ apresentando $\mathrm{R}>0,995$. Os parâmetros LPD e LIRL foram determinados e apresentaram os valores $1,0 \times 10^{-5}$ e $5,0 \times 10^{-4} \mathrm{~mol} \mathrm{~L}^{-1}$, respectivamente. Segundo $\mathrm{Li}$ e colaboradores, ${ }^{12}$ existem algumas vantagens em utilizar este tipo de eletrodo, como a membrana de $\mathrm{Ag}_{2} \mathrm{~S}$ altamente seletiva. Existem ainda outras vantagens; além da membrana que dá ao eletrodo uma resposta muito rápida ser fina, não há necessidade de pré-condicionamento.

O preparo da solução padrão de sulfeto de sódio foi realizado de dois modos diferentes: o primeiro, em uma solução de hidróxido de sódio $0,5 \mathrm{~mol} \mathrm{~L}^{-1}$ e o segundo, na solução TAA em pH 13,6. A grande vantagem de utilizar a solução com $\mathrm{pH}$ elevado é que as espécies de enxofre existentes na forma de $\mathrm{S}^{2-}$ estão menos sujeitas às perdas por meio de sua volatilização, se comparado com outras espécies de enxofre que existem a $\mathrm{pH}$ mais baixos, tais como HS ${ }^{-}$ou $\mathrm{H}_{2} \mathrm{~S}$. Como mostrado na Figura 3a, a concentração dos sulfetos permaneceu relativamente estável em TAA em períodos de até 30 dias, enquanto a concentração de sulfetos em hidróxido de sódio diminuiu $83,8 \%$ de sua concentração original em poucos dias, Figura 3b. Foram realizadas determinações analíticas de $\mathrm{S}^{2-}$ de modo a verificar a concentração dessa espécie nas soluções. $\mathrm{O}$ estudo empregando a solução de hidróxido de sódio foi conduzido com dificuldade, devido à alta instabilidade das espécies de sulfeto nesse meio.

Esse tipo de evidência também foi observado por Florence e Farrar, ${ }^{13}$ que observaram que soluções de enxofre de concentração $5 \times 10^{-4} \mathrm{~mol} \mathrm{~L}^{-1} \mathrm{em} 1 \mathrm{~mol} \mathrm{~L}^{-1}$ de hidróxido de sódio, contidas em frascos fechados, perdiam $15 \%$ de enxofre em aproximadamente $40 \mathrm{~min}$. Da mesma forma, Sekerka e Lechner ${ }^{13}$ também observaram, com o passar do tempo, a diminuição da concentração de enxofre em meio alcalino. Uma concentração inicial de $10^{-2} \mathrm{~mol} \mathrm{~L}^{-1}$ resultava em uma concentração final de $10^{-6} \mathrm{~mol} \mathrm{~L}^{-1}$. De acordo com Florence, ${ }^{12,13}$ a oxidação do enxofre em soluções alcalinas ocorre de forma rápida e quantitativa.

A estabilidade da solução padrão de sulfeto em TAA é mostrada na Figura 3a. Segundo esse resultado, optou-se pelo uso dessa solução para a coleta do SVA. A solução de TAA consiste de um tampão que mantém o pH da solução em um nível alcalino com alta força iônica e a presença do ácido ascórbico retarda a oxidação do íon sulfeto em solução. ${ }^{7,12} \mathrm{O}$ nível alcalino com alta força iônica favorece o deslocamento do equilíbrio no sentido de formação do sulfeto. Baseado no estudo de constantes de equilíbrio de ionização pode-se dizer que a concentração do íon $\mathrm{S}^{2-}$ em solução pode ser ajustada regulando-se a concentração do íon $\mathrm{H}^{+}$. $\mathrm{O}$ aumento da concentração de íons $\mathrm{OH}^{-}$ faz deslocar o equilíbrio e aumentar a concentração dos íons HS e, também, dos íons $\mathrm{S}^{2-}$ e assim dissolver uma quantidade adicional do gás $\mathrm{H}_{2} \mathrm{~S}$ no meio líquido. ${ }^{14} \mathrm{O}$ gás $\mathrm{H}_{2} \mathrm{~S}$ em solução é uma mistura de $\mathrm{H}_{2} \mathrm{~S}$, HS e $^{2-}$ e o equilíbrio químico é dependente do $\mathrm{pH}$ da solução em que $\mathrm{O}_{2} \mathrm{~S}$ se dissolve. ${ }^{12}$

Na literatura são citadas soluções semelhantes para a determinação de sulfetos. Calzada ${ }^{12}$ preparou uma solução contendo hidróxido de sódio, ácido ascórbico e $\mathrm{Na}_{2}$ (EDTA) para determinação de sulfeto em amostras sólidas, utilizando um eletrodo íon-seletivo. Concluiu que essa metodologia é eficiente em concentrações abaixo de $10^{-3}$ mol L ${ }^{-1}$. Já Christensen e colaboradores ${ }^{6}$ utilizaram a mesma solução para determinar sulfetos em amostras de sedimento marinho, utilizando um eletrodo íon-seletivo. Esses autores observaram que a concentração de sulfeto no controle, contendo a solução de TAA, era diversas ordens de grandeza mais baixa que as concentrações de interesse, podendo, desta forma, ser negligenciada. Neste trabalho as quantidades de sulfetos contidas nas soluções de controle também podem ser consideradas negligenciáveis.

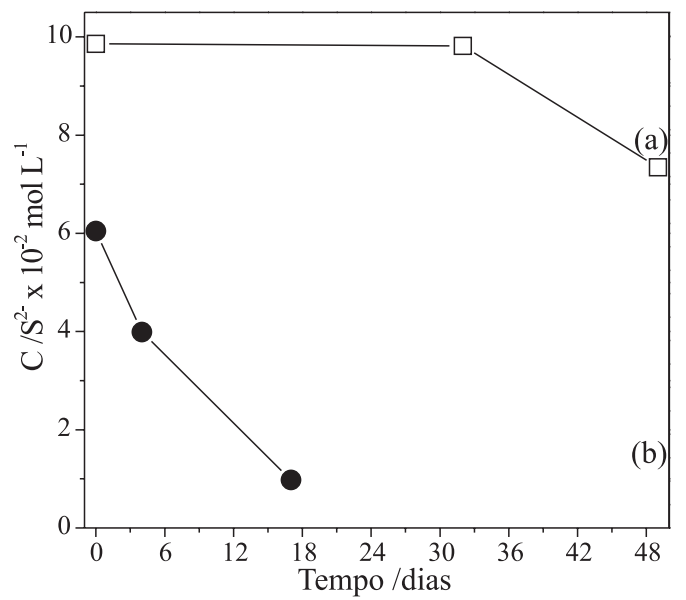

Figura 3. (a) Estabilidade da solução padrão de sulfeto em TAA, pH 13,6. (b) Estabilidade da solução padrão de sulfeto em $\mathrm{NaOH} \mathrm{0,5} \mathrm{mol} \mathrm{L}^{-1}$

Um planejamento fatorial foi empregado para avaliar simultaneamente as variáveis significativas e indicar as melhores condições experimentais no sistema de extração e coleta do SVA com um número reduzido de ensaios experimentais. A Tabela 1 mostra os fatores e seus níveis. A justificativa para a escolha dos fatores temperatura, tempo de extração, solução de TAA e vazão de gás $\mathrm{N}_{2}$ está em obter a melhor eficiência no procedimento analítico de extração e coleta do SVA. Esses fatores foram escolhidos baseados em experimentos preliminares e dados obtidos na literatura. ${ }^{4}$

Tabela 1. Planejamento fatorial $2^{4}$ fatores e níveis

\begin{tabular}{lcc}
\hline Fatores & Menor nível (-) & Maior nível (+) \\
\hline A - Temperatura & ambiente & $0{ }^{\circ} \mathrm{C}$ \\
B - Tempo de extração & $30 \mathrm{~min}$ & $60 \mathrm{~min}$ \\
C - Solução TAA & $100 \mathrm{~mL}$ & $150 \mathrm{~mL}$ \\
D - Vazão de gás $\mathrm{N}_{2}$ & $40 \mathrm{~cm}^{3} \mathrm{~min}^{-1}$ & $90 \mathrm{~cm}^{3} \mathrm{~min}^{-1}$ \\
\hline
\end{tabular}

A Tabela 2 mostra a matriz utilizada na organização do planejamento fatorial dos 16 experimentos contendo o código dos efeitos da matriz para as quatro variáveis em dois níveis, maior e menor (+, -); e os resultados correspondem aos potenciais (em $\mathrm{mV}$ ) para a espécie sulfeto.

A partir desses resultados, a análise estatística foi feita através do programa computacional Fatorial ${ }^{\circledR}$, que gera gráficos normais de estimativas. A Figura 4 descreve esse tipo de gráfico e apresenta os resultados dos cálculos dos efeitos obtidos para cada uma das respostas de interesse. Os efeitos de pequena magnitude, que não são significativos, possuem distribuição normal com média zero e variância constante e tendem a estar distribuídos ao longo de uma reta. Já os efeitos significativos possuem médias diferentes de zero e, portanto, devem estar afastados dessa reta. ${ }^{5,15}$ 
Tabela 2. Matriz de 16 experimentos. Código dos efeitos para as variáveis em dois níveis e os resultados em mV

\begin{tabular}{|c|c|c|c|c|c|c|}
\hline Ensaios & Fator A & Fator B & Fator C & Fator D & Código dos efeitos & Resultados (mV) \\
\hline 1 & - & - & - & - & I & $-742,8$ \\
\hline 2 & + & - & - & - & A & $-744,4$ \\
\hline 3 & - & + & - & - & B & $-752,0$ \\
\hline 4 & + & + & - & - & $\mathrm{AB}$ & $-752,8$ \\
\hline 5 & - & - & + & - & $\mathrm{C}$ & $-741,0$ \\
\hline 6 & + & - & + & - & $\mathrm{AC}$ & $-750,1$ \\
\hline 7 & - & + & + & - & $\mathrm{BC}$ & $-750,8$ \\
\hline 8 & + & + & + & - & $\mathrm{ABC}$ & $-749,1$ \\
\hline 9 & - & - & - & + & $\mathrm{D}$ & $-753,2$ \\
\hline 10 & + & - & - & + & $\mathrm{AD}$ & $-754,3$ \\
\hline 11 & - & + & - & + & $\mathrm{BD}$ & $-755,4$ \\
\hline 12 & + & + & - & + & $\mathrm{ABD}$ & $-754,5$ \\
\hline 13 & - & - & + & + & $\mathrm{CD}$ & $-753,0$ \\
\hline 14 & + & - & + & + & $\mathrm{ACD}$ & $-753,5$ \\
\hline 15 & - & + & + & + & $\mathrm{BCD}$ & $-753,8$ \\
\hline 16 & + & + & + & + & $\mathrm{ABCD}$ & $-752,2$ \\
\hline
\end{tabular}

Analisando a Figura 4, verifica-se que a melhor condição experimental corresponde ao experimento 11 que relaciona ambos os fatores, $\mathrm{B}$ e D, devido ao maior valor absoluto do efeito. Os resultados obtidos nos experimentos 1 a 8 , que foram aqueles realizados com menor vazão de $\mathrm{N}_{2}$, indicaram menor taxa de recuperação para os sulfetos, uma vez que o $\mathrm{H}_{2} \mathrm{~S}$ permaneceu no balão de reação, devido à baixa vazão do gás, sendo que a vazão de $\mathrm{N}_{2}$ é diretamente dependente do arranjo do sistema de extração. No tempo de extração de $30 \mathrm{~min}$, nem todo o sulfeto é liberado do balão de reação. Portanto, pode-se observar que o efeito da interação das variáveis tempo de extração (B) e vazão de $\mathrm{N}_{2}$ (D) é significativo.

Em relação ao estudo da temperatura, não houve uma influência significativa no sistema de extração e coleta. Este procedimento de avaliação da temperatura foi realizado para verificar a interação na solubilidade do $\mathrm{H}_{2} \mathrm{~S}$, uma vez que esse é muito volátil. Desta maneira, poderiam ser evitadas possíveis perdas. Acredita-se que este comportamento não foi observado devido à eficiência da solução TAA.

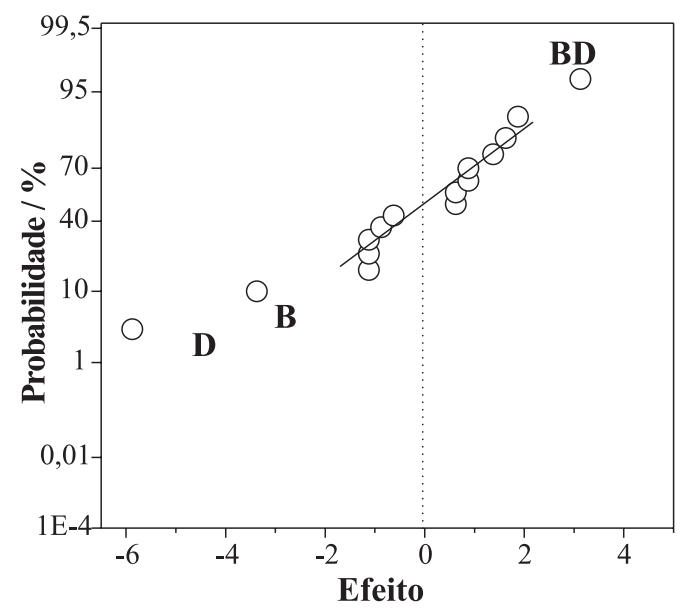

Figura 4. Gráfico normal de estimativas com os resultados dos cálculos dos efeitos para cada uma das respostas
A partir dos resultados obtidos no planejamento fatorial foram realizados testes de recuperação para uma solução padrão de sulfeto de sódio. Os resultados são mostrados na Tabela 3. Foram testadas concentrações na mesma ordem de grandeza dos limites LPD e LIRL. A porcentagem de recuperação dos sulfetos variou de 93 a 116\%. Esses resultados são consistentes com valores descritos na literatura ${ }^{16}$ que citam a recuperação para sulfetos em águas na ordem de 80 a $120 \%$.

Tabela 3. Estudo da recuperação de sulfetos no sistema de extração, $\mathrm{n}=3$

\begin{tabular}{lcc}
\hline $\begin{array}{l}\text { C adicionada de } \\
\mathrm{S}^{-2} / \mathrm{mol} \mathrm{L}^{-1}\end{array}$ & $\begin{array}{c}\text { C recuperada de } \\
\mathrm{S}^{-2} / \mathrm{mol} \mathrm{L}^{-1}\end{array}$ & Recuperação (\%) \\
\hline $1 \times 10^{-4}$ & $1,05 \times 10^{-4} \pm 7,2 \times 10^{-6}$ & $103-116$ \\
$1 \times 10^{-5}$ & $1,05 \times 10^{-5} \pm 8,8 \times 10^{-7}$ & $93-114$ \\
\hline
\end{tabular}

Segundo Mueller e colaboradores, ${ }^{17}$ quando trabalharam com baixas concentrações de sulfeto, essas foram susceptíveis à oxidação pelo ar principalmente em elevado $\mathrm{pH}$, ocorrendo efeitos de sorção no corpo do eletrodo e nas paredes da célula eletroquímica. $\mathrm{O}$ mesmo não foi observado em nosso estudo. Acredita-se que estes resultados foram possíveis devido aos cuidados, principalmente com o meio inerte e a solução TAA.

As concentrações de sulfeto em estudo foram obtidas através do procedimento gráfico fundamentado na Equação 1:

$\left(V_{o}+V_{S}\right) 10^{E / S}=10^{K / S} V_{0} C_{X}+10^{K / S} C_{S} V_{S}$

onde: $V_{0}=$ volume inicial da amostra de concentração desconhecida; $V_{S}=$ volume do padrão adicionado; $E=$ leitura em volts do potencial; $K=$ constante característica do eletrodo; $C_{X}=$ concentração desconhecida na amostra; $C_{S}=$ concentração do padrão

$$
S=(\beta R T / n F) \ln 10
$$

onde: $\beta=$ constante de valor igual a $1 ; R=$ constante dos gases; 
$T=$ temperatura $(K) ; n=$ carga do íon a ser detectado $F=$ constante de Faraday $\left(9,64853415 \times 10^{4} \mathrm{C} \mathrm{mol}^{-1}\right)$.

A partir da Equação 1 um gráfico de $\left(\mathrm{V}_{0}+\mathrm{V}_{\mathrm{S}}\right) 10^{\mathrm{E} / \mathrm{S}}$ contra $\mathrm{VS}_{\mathrm{S}}$ gera uma reta com coeficiente angular $(m)$ igual a $10^{\mathrm{K} / \mathrm{s}} \mathrm{C}_{\mathrm{S}}$ e uma interseção com o eixo $\mathrm{x}$ igual a $\mathrm{V}_{0} \mathrm{C}_{\mathrm{x}}$. A interseção é obtida fazendo-se $\mathrm{y}=0 .{ }^{18} \mathrm{Na}$ Figura 5 apresenta-se o gráfico de adição de padrão para uma solução $1 \times 10^{-5} \mathrm{~mol} \mathrm{~L}^{-1}$. A reta apresentou um coeficiente linear $\mathrm{R}$ igual a 0,99995. A interseção apresentou um valor de $0,0344 \mathrm{~mL}$ resultando em uma concentração de sulfeto de $9,3 \times 10^{-6} \mathrm{~mol} \mathrm{~L}^{-1}$. A recuperação foi de $93 \%$.

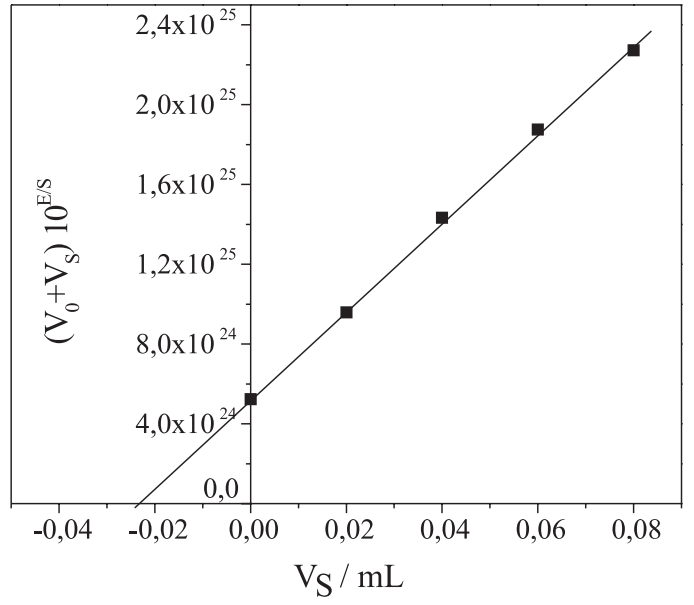

Figura 5. Gráfico de adição de padrão para o teste de recuperação; $n=3$

Definidas as melhores condições experimentais, foi realizada a aplicação com as amostras de sedimentos coletados nos Rios Iraí e Iguaçu. Foram extraídos e coletados na solução TAA $2,41 \times 10^{-5}$ e $5,87 \times 10^{-4} \mathrm{~mol} \mathrm{~L}^{-1}$ de sulfeto de sedimentos dos Rios Iraí e Iguaçu, respectivamente. A Figura 6 mostra os resultados obtidos para a determinação do SVA nas amostras.

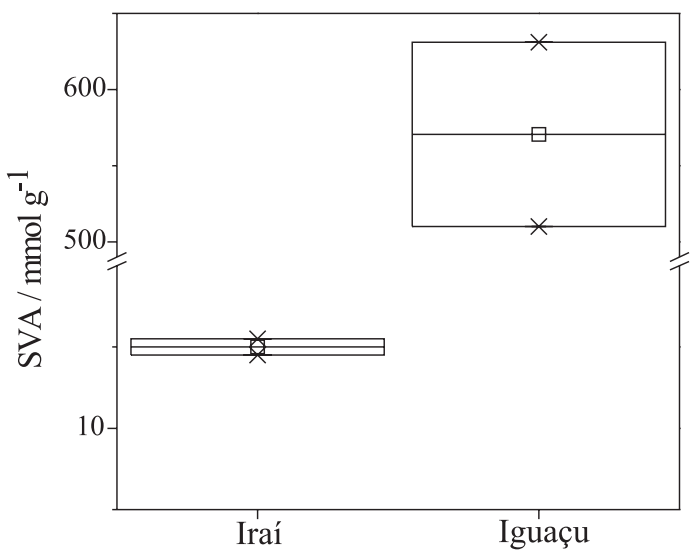

Figura 6. Valores de SVA para as amostras de sedimento dos Rios Iraí e Iguaçu. As linhas horizontais da caixa representam 25, 50 e 75\% dos valores; barras de erro indicam 5 e 95\%; (X) expressa 1 e 99\% e (-) corresponde aos valores máximos e mínimos. A média aritmética é representada pelo símbolo $(\square)$

Para uma amostra de sedimento do Rio Iguaçu com massa seca igual a 4 g obteve-se uma média de $570,5 \mu \mathrm{mol} \mathrm{g} \mathrm{g}^{-1}$ de SVA, com um desvio padrão de $85,56 \mu \mathrm{mol} \mathrm{g}{ }^{-1}$ e um coeficiente de variação de $15,00 \%$. Para a amostra de 7,0 g de sedimento seco do Rio Iraí obteve-se 11,0 $\mu$ mol g ${ }^{-1}$ de SVA, em média, com um desvio padrão de $0,14 \mu \mathrm{mol} \mathrm{g}^{-1}$ e um coeficiente de variação de $1,28 \%$. Os resultados do coeficiente de variação apresentaram-se menores ou iguais a $15 \%$. Portanto, pode-se concluir que se trata de conjunto de dados homogêneo e estável de baixa dispersão.

Os resultados obtidos para SVA evidenciam que esses sedimentos possuem características diferentes, sendo representativos de ambientes bastante distintos. Enquanto o Rio Iraí tem características de um corpo aquático menos impactado, o Rio Iguaçu apresenta níveis elevados de diversos poluentes ambientais, especialmente aqueles associados ao descarte de esgoto. ${ }^{10,19}$

\section{CONCLUSÕES}

O protocolo experimental mostrou-se eficiente quanto à extração do SVA. O estudo quimiométrico revelou que os fatores vazão do gás $\mathrm{N}_{2}$ e tempo de extração são as variáveis mais importantes de todo o sistema. A fixação da espécie $\mathrm{S}^{2-}$ em solução só foi possível com a utilização da solução TAA.

A determinação da espécie $\mathrm{S}^{2-}$ foi feita pelo método potenciométrico, que permitiu trabalhar com baixas concentrações. Testes de recuperação mostraram respostas satisfatórias na ordem de 93 a $116 \%$ para concentrações de $\mathrm{S}^{2-}$ variando entre $1 \times 10^{-5}$ e $1 \times 10^{-4} \mathrm{~mol} \mathrm{~L}^{-1}$.

Os valores médios de SVA para as amostras de sedimento dos Rios Iraí e Iguaçu foram, respectivamente, 11,0 e $570,5 \mu \mathrm{mol} \mathrm{g}^{-1}$ com base no peso seco de sedimento. Esta diferença dos valores de SVA pode ser atribuída às diferentes características destes rios. A informação acerca do SVA tem sido considerada crucial para uma compreensão do impacto ambiental associado à especiação química de metais em sedimentos, pois uma previsão mais realista da toxicidade de sedimentos leva em consideração a comparação entre as determinações dos metais e o SVA.

\section{AGRADECIMENTOS}

Ao CNPq/CT-HIDRO e à Fundação Araucária (PR) pelo apoio financeiro. A F. F. Sodré (Instituto de Química, UNICAMP) e N. F. Leite (Instituto de Tecnologia do Paraná) pelo auxílio na coleta das amostras de sedimento.

\section{REFERÊNCIAS}

1. http://www.sednet.org, acessada em Abril 2007; Yu, K. C.; Tsai, L. J.; Chen, S. H.; Ho, S. T.; Wat. Res. 2001, 35, 4086.

2. Billon, G.; Ouddane, B.; Recourt, P.; Boughriet, A.; Estuar. Coast. Shelf Sci. 2002, 55, 167.

3. Stén, P.; Forsling, W. A.; Colloids Surf. 2000, 172, 17; Simpson, S. L.; Apte, S. C.; Batley, G. E.; Environ. Sci. Technol. 2000, 34, 4533.

4. Allen, H. E.; Fu, G.; Deng, B.; Environ. Toxicol. Chem. 1993, 12, 1441; Di Toro, D. M.; Mahony, J. D.; Hansen, D. J.; Scott, K. J.; Hicks, M. B.; Mayr, S. M.; Redmond, M.; S. Environ. Toxicol. Chem. 1990, 9, 1487.

5. He, Y.; Zheng, Y.; Locke, D. C.; Anal. Chim. Acta. 2002. 459, 209; Sodré, F. F.; Anjos, V. E.; Prestes, E. C.; Grassi, M. T.; J. Environ. Monit. 2005, 7, 581; Barros Neto, B.; Scarminio, I. S.; Bruns, R. E.; Como fazer Experimentos: Pesquisa e Desenvolvimento na Ciência e na Indústria, 2a ed., UNICAMP: Campinas, 2002; Barros Neto, B.; Scarminio, I. S.; Bruns, R., E.; Quim. Nova. 2006, 29, 1401; Neves, C. F. C.; Schvartzman, M. M. A. M.; Quim. Nova. 2005, 28, 622; Costa, L. M.; Korn, M. G. A.; Castro, J. T.; Santos, W. P. C.; Carvalho, E. V.; Nogueira, A. R. A.; Quim. Nova. 2006, 29, 149.

6. Christensen, J. K.; Høyer, B.; Kryger, L.; Pind, N.; Kong, L. S.; J. Chem. Educ. 1998, 75, 1605.

7. Hassan, S. S. M.; Marzouk, S. A. M.; Sayour, H. E. M.; Anal. Chim. Acta 2002, 466, 47. 
8. Silva, S. A.; Dissertação de Mestrado, Universidade Federal de Santa Catarina, Brasil, 2000.

9. He, Y.; Zheng, Y.; Locke, D. C.; Anal. Chim. Acta. 2002, 459, 209.

10. Superintendência de Desenvolvimento de Recursos Hídricos e Saneamento Ambiental; Qualidade das águas interiores do Estado do Paraná: 1987-1995, Curitiba, 1997; Scheffer, E. W.; Sodré, F. F.; Grassi, M. T.; Quim. Nova. 2007, 30, 332.

11. Campos, M. L. A. M.; Bendo, A.; Viel, F. C.; Quim. Nova. 2002, 25, 808.

12. Li, G.; Polk, J. B.; Meazell, L. A.; Hatchett, D. W.; J. Chem. Educ. 2000, 77, 1049; Su, Y. S.; Cheng, K. L.; Jean, Y. C.; Talanta. 1997, 44, 1757; Calzada, M. G.; Marbán, G.; Fuertes, A. B.; Anal. Chim. Acta 1999, 380, 39; Balasubramanian, S.; Pugalenthi, V.; Wat. Res. 2000, 34, 17, 4201.

13. Florence, T. M.; Farrar, Y. J.; Anal. Chim. Acta 1980, 116, 175; Sekerka, Y. J.; Lechner, F.; Anal. Chim. Acta 1977, 93, 139; Florence, T. M.; J. Electroanal. Chem. 1979, 41, 219.
14. Quagliano, J. V.; Vallarino, L. M.; Química, 3ª ed.; Guanabara dois: Rio de Janeiro, 1979.

15. Werkema, M. C. C.; Aguiar, S.; Otimização Estatística de Processos: Como Determinar a Condição de Operação de um Processo que Leva ao Alcance de uma Meta de Melhoria; Série Ferramentas da Qualidade, Fundação Christiano Ottoni: Belo Horizonte, 1996.

16. Clesceri, L. S.; Greenberg, A. E.; Trussell, R.R.; ASTM-APHA. Standard Methods for the Examination of Water and Wastewater, $17^{\text {th }}$ ed., M.A.H. Eds: Washington, DC, 1989.

17. Muller, B.; Stierli, R.; Anal. Chim. Acta 1999, 401, 257.

18. Harris, D. C.; Análise Química Quantitativa, $6^{\mathrm{a}}$ ed.; LTC- Livros Técnicos e Científicos Editora S.A., 2005.

19. Sodré, F. F.; Grassi, M. T. ; Water, Air, Soil Pollut. 2007, 178, 103. 